\title{
Otimização da atividade de herbicidas com diferentes adjuvantes na fitotoxicidade de Digitaria insularis no estádio de florescimento
}

Arthur Duarte Rodrigues Neto, Matheus da Silva Almeida, Evandro Pereira Prado, Leandro Tropaldi, Ana Carolina Firmino, Carolina dos Santos Batista Bonini, Ronaldo da Silva Viana, Ronaldo Cintra Lima

Universidade Estadual Paulista - UNESP - FCAT, Dracena, SP. E-mail: arthurodrigues@gmail.com

\section{Resumo}

O presente trabalho teve como objetivo quantificar o efeito proporcionado pela interação entre os herbicidas clethodim e paraquat associados com diferentes classes de adjuvantes na fitotoxicida de de Digitaria insularis no estádio de florescimento. Os herbicidas foram aplicados nas doses de $0 ; 6,25 ; 12,5$; 25; 50; 75 e $100 \%$ da recomendada pelo fabricante, de forma isolada e em mistura com os adjuvantes: nonil fenoxi poli (etilenoxi) etanol $(0,05 \%)$; copolímero de poliéter e silicone $(0,1 \%)$; lecitina e ácido propiônico $(0,5 \%)$ e óleo mineral $(0,5 \%)$ no delineamento inteiramente casualizado com cinco repetições em esquema fatorial (doses dos herbicidas $x$ adjuvantes). 0 efeito fitotóxico foi visualmente avaliado aos 12 dias após aplicação (DAA) para o herbicida paraquat e aos 21 DAA para o herbicida clethodim. Verificou-se em plantas de $D$. insularis fitotoxidade potencializada para o herbicida clethodim nos tratamentos com os adjuvantes nonil fenoxi poli (etilenoxi) etanol, lecitina e ácido propiônico e óleo mineral. Para o herbicida paraquat, a potencialização da fitotoxicidade nas plantas de $D$. insularis foi proporcionada somente pelo tratamento com o adjuvante lecitina e ácido propiônico.

Palavras-chave: tecnologia de aplicação de defensivos; capim-amargoso; controle químico; planta daninha.

Optimizing activity of herbicides with different adjuvant on phytotoxicity of Digitaria insularis at flowering stage

\begin{abstract}
The aim of this paper was quantify the effect of the herbicides clethodim and paraquat associated with different adjuvants class on phytotoxicity of Digitaria insularis at flowering stage. The herbicides were applied at $0,6.25,12.5,25,50,75$ and $100 \%$ of recommended label isolated or associated with the adjuvants: nonylphenoxy polyethoxy ethanol at $0.05 \%$; silicone polyether copolymer at $0.1 \%$; lecithin-based adjuvant and acid propionic at $0.5 \%$ and mineral oil at $0.5 \%$. The experimental design was completely randomized with five replications in factorial scheme (herbicides rates $\mathrm{x}$ adjuvants). The phytotoxicity effect was visually assessed for paraquat at 12 days after treatment (DAT) and at 21 DAT for clethodim. The treatments applied clethodim with the adjuvants nonylphenoxy polyethoxy ethanol, lecithin-based adjuvant and acid propionic and mineral oil significantly increased the phytotoxicity of $D$. insularis plants. For the paraquat the increase phytotoxicity of $D$. insularis plants was provided by the treatment with the lecithin-based and acid propionic adjuvant.
\end{abstract}

Keywords: pesticide application technology; sourgrass; chemical control; weed.

\section{Introdução \\ O capim-amargoso (Digitaria insularis (L.)} Fedde) é uma espécie gramínea, herbácea, perene que se reproduz por sementes e/ou rizomas. Essa planta é considerada uma das plantas daninhas mais importantes do Brasil, frequentemente encontrada infestando culturas perenes como pastagens, cafezais, pomares, grandes culturas como soja, milho, algodão, além de áreas destinadas para produção de hortaliças 
nas proximidades de centros urbanos e áreas não agrícolas, como beira de estradas e terrenos baldios, por exemplo (MACHADO et al., 2008; CARVALHO et al., 2011).

O surgimento de cultivares tolerantes ao herbicida glyphosate favoreceu o uso intensivo e exclusivo desse herbicida em diversos sistemas produtivos. Com isso, o controle de capimamargoso com glyphosate tem sido cada vez menos eficiente, ao passo que os relatos de biótipos com resistência a esse herbicida são cada vez maiores (LOPEZ-OVEJERO et al. 2017). Atualmente, o manejo de capim-amargoso é um dos grandes desafios para a agricultura brasileira.

O desempenho de alguns herbicidas pode ser incrementado com o uso de adjuvantes, uma vez que essas substâncias podem propiciar o aumento da concentração do ingrediente ativo retido no alvo e o aumento da quantidade absorvida pela superfície da planta (HOLLOWAY et al., 2000). Portanto, muitas vezes a correta seleção e adição de adjuvantes pode ser uma forma de aumentar o desempenho de herbicidas e de minimizar seu impacto em organismos não alvo (LI et al., 2016). Além disso, alvos verticais, como as gramíneas, possuem menor superfície disponível para a captura das gotas, comprometendo sua retenção, comparando com alvos horizontais (MASSINON et al., 2014).

Para o manejo de áreas com predominância de ocorrência de capimamargoso, herbicidas alternativos ao glyphosate, como o paraquat e clethodim são frequentemente utilizados (MELO et al., 2012). Para ambos defensivos, os fabricantes recomendam a adição de adjuvantes para obtenção de melhores resultados, mas devido as diferentes modalidades (classes/grupos) de adjuvantes disponível no mercado, ainda existem pouca informação sobre os efeitos dos mesmos.

O emprego de adjuvantes surfatantes na calda de pulverização tem habilidade de diminuir a tensão superficial da gota, aumentar a molhabilidade da cutícula pelo maior espalhamento resultando em aumento da quantidade e qualidade depositada (HOLLOWAY et al., 2000; RYCKAERT et al., 2007; DORR et al., 2016).

O controle de capim-amargoso em estádio avançado (3 a 5 perfilhos com $30-40 \mathrm{~cm}$ de altura) não é satisfatório quando o clethodim é utilizado de forma isolada (108 g de i.a ha ${ }^{-1}$ ) (MELO et al., 2012). Zobiole et al. (2016) ressaltam a eficácia dos herbicidas inibidores da
ACCase no controle do capim-amargoso em estádios iniciais de desenvolvimento e, reforçam que, para o controle eficiente (>90\%) em estádios mais avançados como o de florescimento, são necessárias aplicações sequenciais ou associação de produtos para o controle desta espécie, sendo o capim-amargoso mais sensível à aplicação de herbicidas até o 35으 dia pós emergência, antes da formação dos rizomas (MACHADO et al., 2006; PEREIRA et al., 2010).

Para herbicidas de ação local com baixa translocação como o paraquat, um fator relevante para a expressão de seus efeitos é a uniformidade da cobertura pela pulverização. Quanto maior a uniformidade de cobertura pela calda pulverizada, maior a eficiência do herbicida de contato (VARGAS; ROMAN, 2006). O perfilhamento do capim-amargoso dificulta a penetração da aplicação no interior da planta e impede que o herbicida atinja a planta toda, fator essencial para o sucesso da ação de herbicidas de contato como o paraquat, ocasionando a rebrota posterior por não atingir o rizoma (ZOBIOLE et al. 2016).

Nesse contexto, a utilização de adjuvantes em mistura com herbicidas pós-emergentes pode ser uma importante ferramenta para aumentar a atividade biológica desses agentes e que pesquisas visando o entendimento da interação entre adjuvantes, herbicidas e planta daninha podem contribuir para o aumento da eficiência de controle. Portando, o objetivo do trabalho foi verificar a fitotoxicidade do capim-amargoso em estádio avançado de desenvolvimento (florescimento), proporcionada pela interação dos herbicidas clethodim e paraquat em mistura com diferentes classes de adjuvantes.

\section{Materiais e Métodos}

Dois experimentos de curva de doseresposta foram conduzidos, um para o herbicida clethodim (Select ${ }^{\circledR} 240$ EC) e outro para paraquat (Gramoxone ${ }^{\circledR}$ 200). Ambos experimentos foram conduzidos em casa de vegetação, entre os meses de fevereiro e abril de 2018, no município de Dracena, no campus da UNESP/FCAT Faculdade de Ciências Agrárias e Tecnológicas Campus de Dracena -SP, com coordenadas geográficas, $21^{\circ} 29^{\prime}$ de Latitude Sul e $51^{\circ} 52^{\prime}$ de Longitude Oeste. Os herbicidas foram aplicados nas concentrações de $0 ; 6,25 ; 12,5 ; 25$; $50 ; 75$ e $100 \%$ da dose recomendada pelo fabricante (108 g i.a. ha ${ }^{-1}$ de clethodim e $400 \mathrm{~g}$ i.a. $\mathrm{ha}^{-1}$ de paraquat) de forma isolada (T1) e em 
mistura com os adjuvantes: nonil fenoxi poli (etilenoxi) etanol $\left(\mathrm{Agral}^{\oplus}\right)$ na concentração de 0,05\% (T2); copolímero de poliéter e silicone (Silwet ${ }^{\circledR}$ ) a 0,1\% (T3); lecitina e ácido propiônico (LI700 ${ }^{\circledR}$ ) a $0,5 \%$ (T4) e óleo mineral (Nimbus ${ }^{\circledR}$ ) a $0,5 \%$ (T5). Os experimentos foram realizados no delineamento experimental inteiramente casualizado com cinco repetições em esquema fatorial 7 (doses de herbicida) $\times 4$ (classes de adjuvantes) +1 tratamento testemunha (somente herbicida).

Para isso, as sementes de capim-amargoso foram semeadas em vasos de $1,7 \mathrm{~L}$ de capacidade a $1 \mathrm{~cm}$ de profundidade e desbastadas mantendo duas plantas por vaso, sendo conduzidas em casa de vegetação até o início do florescimento (35 dias após emergência), momento em que os tratamentos foram aplicados.

As aplicações foram realizadas utilizandose um pulverizador costal (Herbicat ${ }^{\circledR}$ ) pressurizado por $\mathrm{CO}_{2}$, equipado com 4 pontas de jato plano XR 8002 na pressão de 200 kPa (vazão: $\left.0,65 \mathrm{~L} \mathrm{~min}^{-1}\right)$, espaçadas em $0,5 \mathrm{~m}$, com velocidade de deslocamento do aplicador de aproximadamente $5 \mathrm{~km} \mathrm{~h}^{-1}$, altura de barra de $0,75 \mathrm{~m}$ em relação ao topo das plantas e volume de aplicação de aproximadamente $156 \mathrm{~L} \mathrm{ha}^{-1}$. A aplicação foi realizada dentro de uma casa de vegetação evitando o efeito de rajadas de vento na temperatura de $28 \pm 2^{\circ} \mathrm{C}$, com velocidade de vento inferior a $2,4 \mathrm{~km} \mathrm{~h}^{-1}$ e umidade relativa do ar de $59 \pm 5 \%$.

Após a aplicação, os vasos permaneceram em casa de vegetação, sem irrigação por 12 horas a fim de garantir a absorção dos herbicidas e, após esse período, irrigadas diariamente via aspersão conforme necessidade das plantas. As avaliações visuais de fitotoxicidade foram realizadas aos 12 dias após aplicação (DAA) do herbicida paraquat e aos 21 DAA do herbicida clethodim. Essa avaliação foi baseada na proposta da SBCPD (1995), considerando como zero para ausência de sintomas e $100 \%$ a morte da planta.

As análises da resposta de porcentagem de fitotoxicidade das plantas de capim-amargoso pela aplicação do herbicida com e sem adjuvante foram ajustadas pelo modelo log-logístico proposto por Seefeldt et al. (1995) como descrito conforme equação 1 .

$Y=\frac{D-C}{1+\exp \{b[\log (X)-\log (C 50)]\}}+C$

Em que: $Y=$ resposta da porcentagem de fitotoxicidade; $X=$ dose do herbicida; $D=$ limite superior da curva; $C=$ limite inferior da curva; $b=$ declividade da curva; $C_{50}=$ dose necessária para proporcionar $50 \%$ de fitotoxicidade em relação às plantas sem aplicação do herbicida (controle).

As análises das curvas de dose-resposta e os valores de $C_{50}$ foram determinados utilizando o pacote drc no software R (RITZ et al., 2015). Foi utilizado o teste lack-of-fit para verificar se houve bom ajuste da curva utilizando o modelo de quatro parâmetros sendo aceito quando o valor de $P \geq 0,05$. Os valores de $C_{50}$ dos tratamentos foram comparados pelo intervalo de confiança a 95\% de probabilidade.

\section{Resultados e Discussão}

$O$ teste $F$ sobre a análise da variância demonstrou significância da interação fatorial entre herbicidas e doses $(p<0,01)$. De acordo com o teste lack-of-fit ao nível de $95 \%$ não foi significativo para nenhum herbicida e curvas testadas, indicando que o modelo proposto se ajustou de forma satisfatório. As estimativas dos parâmetros da equação log-logística encontramse descritos na Tabela 1. 
Tabela 1. Estimativas dos parâmetros $B, C$ e D do modelo log-logístico ${ }^{a}$, que descrevem a fitotoxicidade visual de capim-amargoso (Digitaria insularis) no estádio de florescimento, quando submetidos a diferentes doses dos herbicidas clethodim e paraquat e classes de adjuvantes.

\begin{tabular}{lcccc}
\hline Tratamento & $B$ & $C$ & $D$ & $C_{50}{ }^{b}$ \\
\hline Clethodim & $-1,38$ & $-0,7$ & 82,3 & 107,1 \\
Clethodim + nonil fenoxi poli (etilenoxi) etanol & $-1,23$ & $-0,7$ & 82,3 & 61,2 \\
Clethodim + copolímero de poliéter e silicone & $-0,91$ & $-0,7$ & 82,3 & 81,1 \\
Clethodim + lecitina e ácido propiônico & $-1,49$ & $-0,7$ & 82,3 & 57,1 \\
Clethodim + óleo mineral & $-0,91$ & $-0,7$ & 82,3 & 50,8 \\
Paraquat & $-1,24$ & 7,7 & 96,8 & 260,8 \\
Paraquat + nonil fenoxi poli (etilenoxi) etanol & $-4,68$ & 7,7 & 96,8 & 253,2 \\
Paraquat + copolímero de poliéter e silicone & $-2,42$ & 7,7 & 96,8 & 190,3 \\
Paraquat + lecitina e ácido propiônico & $-1,51$ & 7,7 & 96,8 & 80,3 \\
Paraquat + óleo mineral & $-3,21$ & 7,7 & 96,8 & 181,9 \\
\hline
\end{tabular}

${ }^{a}$ Equação: $Y=\frac{D-C}{1+\exp \{b[\log (X)-\log (C 50)]\}}+C$

${ }^{b} C_{50}=$ dose necessária para proporcionar $50 \%$ de fitotoxicidade visual em relação às plantas controle (sem aplicação de herbicida).

A adição de adjuvante nas caldas, tanto do herbicida clethodim quanto do herbicida paraquat, diminuiu a dose necessária para promover $50 \%$ de fitotoxicidade $\left(C_{50}\right)$ em comparação com 0 herbicida aplicado isoladamente. Verifica-se maior fitotoxicidade visual quando aplicado o herbicida paraquat com aproximadamente $97 \%$ (parâmetro $D$ ) comparada com o herbicida clethodim com $82 \%$ (Tabela 1 ). Essa maior fitotoxicidade está relacionada ao modo de ação do herbicida paraquat que provoca fitotoxicidade nas plantas nas primeiras horas após a aplicação.

Para o herbicida clethodim, o maior valor de $C_{50}$ foi observado na aplicação sem adjuvante (T1), não diferenciando significativamente apenas do tratamento utilizando o adjuvante copolímero de poliéter e silicone (T3) (Figura 1a). Os tratamentos T2, T4 e T5, diferiram estatisticamente do tratamento sem adjuvante (T1), porém não diferiram do tratamento com copolímero de poliéter e silicone (T3) (Figura 1a).

Figura 1. Valores de dose de ingrediente ativo (i.a.) do herbicida clethodim (a) aos 21 dias após aplicação (DAA) e paraquat (b) aos 12 DAA, necessária para proporcionar $50 \%$ de fitotoxicidade visual nas plantas de capim-amargoso.

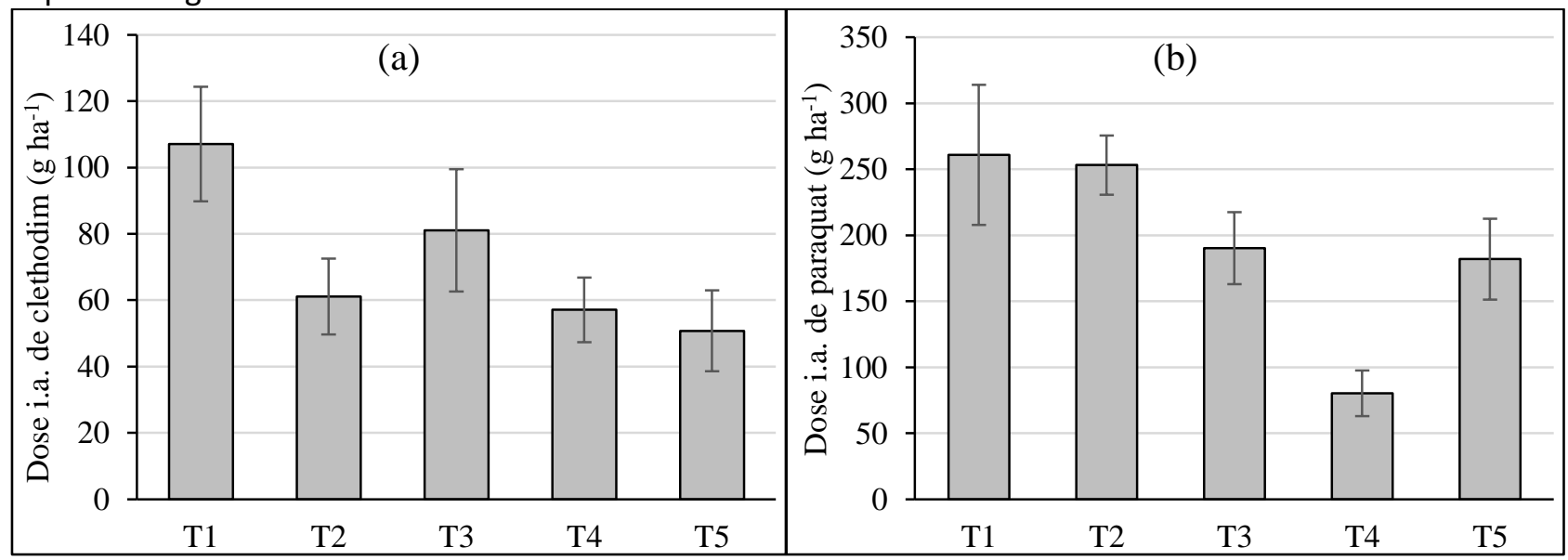

Legenda: T1: herbicida; T2: herbicida + nonil fenoxi poli (etilenoxi) etanol (0,05\%); T3: herbicida + copolímero de poliéter e silicone (0,1\%); T4: herbicida + lecitina e ácido propiônico (0,5\%) e T5: herbicida + óleo mineral $(0,5 \%)$. As barras verticais representam o intervalo de confiança a $95 \%$ de probabilidade.

Jordan et al. (1996), testaram a influência de adjuvantes na eficácia de clethodim no controle de diferentes espécies de monocotiledôneas (Echinochloa crus-galli, Urochloa platyphylla e Sorghum halepense) com a adição de adjuvantes de diferentes grupos 
químicos. Os resultados mostraram maior nível de controle quando se associou clethodim com adjuvantes oleosos como óleo mineral e vegetal metilado. A aplicação de clethodim com os adjuvantes oleosos promoveu controle equivalente ou maior das plantas do que quando aplicado o dobro da dose (140 g i.a. ha ${ }^{-1}$ ) do herbicida combinada com adjuvantes à base de silicone e adjuvante não iônicos convencionais. Tais resultados corroboram com os resultados encontrados neste trabalho, onde a associação do adjuvante óleo mineral (T5) favoreceu a ação do herbicida inibidor da ACCase mais do que quando o herbicida foi associado ao adjuvante siliconado (T3) (Figura 1a). Maior fitotoxicidade de clethodim quando associado com óleo mineral provavelmente está associado a maior absorção e translocação do herbicida por esse adjuvante (Nandula et al., 2007).

Salvalaggio et al. (2018), observaram que adjuvantes oleosos (óleo vegetal a 0,3\% e óleo mineral a $0,6 \%$ ) proporcionaram, respectivamente, espalhamento de calda na face adaxial de plantas de capim-amargoso de 32,5 e $43,8 \%$ maior do que do herbicida testado (glufosinato de amônio) isoladamente. Os autores atribuíram que, possivelmente, tais resultados são provenientes da maior quantidade de ceras na superfície adaxial das folhas. Tal característica, teoricamente, favorece a interação entre a superfície foliar e a calda contendo adjuvante do tipo oleoso e consequentemente a retenção/absorção do ingrediente ativo.

Já com o herbicida paraquat, apenas o adjuvante lecitina e ácido propiônico (T4) diferiu significativamente do tratamento com 0 herbicida aplicado isoladamente (T1), reduzindo consideravelmente o valor $C_{50}$, enquanto os demais adjuvantes não potencializaram significativamente a fitotoxicidade desse herbicida (Figura 1b). Avaliando as características físico-químicas de soluções aquosas com adjuvantes agrícolas, Cunha e Alves (2009) observaram que o adjuvante lecitina e ácido propiônico destacou-se na redução do $\mathrm{pH}$ da solução para 3,4 na dose-cheia $(0,5 \%)$, enquanto o adjuvante não siliconados (noni poli etanol ) elevou o $\mathrm{pH}$ da solução para o valor de 7,5 na dose-cheia $(0,2 \%)$, utilizando água com $\mathrm{pH}$ inicial de 5,43 . Os autores ainda argumentaram que, um $\mathrm{pH}$ mais baixo retarda a taxa de hidrólise sofrida pela molécula do herbicida quando na água, mantendo a superfície das folhas (que possuem $\mathrm{pH}$ neutro) úmida por mais tempo e, consequentemente, favorecendo a absorção do herbicida pela superfície foliar.

A potencialização proporcionada pelo adjuvante lecitina e ácido propiônico, pode estar relacionada com o seu efeito acidificador da calda e sua capacidade de aumentar a condutividade elétrica, prevenindo a hidrólise alcalina do herbicida (que se torna instável em $\mathrm{pH}$ maior que $7,0)$, melhorando a performance por adequar o $\mathrm{pH}$ da solução ao $\mathrm{pH}$ de estabilidade do herbicida $(3,95)$ e influenciando na absorção pelos tecidos vegetais (DEER; BEARD, 2001; CUNHA e ALVES, 2009; CUNHA et al., 2017).

A característica acidificante do adjuvante lecitina e ácido propiônico, provavelmente foi a responsável por elevar o potencial fitotóxico do herbicida paraquat, visto que, os outros adjuvantes testados não apresentam tal propriedade. Nota-se também que, a redução da tensão superficial da calda não aparece como fator predominante na promoção da fitotoxidade do herbicida, visto que, dentre os demais adjuvantes testados, os adjuvantes siliconados aparecem na literatura sendo os produtos que mais promovem a redução da tensão superficial, com valores próximos de 20 $\mathrm{mN} \mathrm{m}^{-1}$ na concentração testada nesse experimento (BAIO et al., 2015; PRADO et al., 2016).

Comparando com outros adjuvantes, o tratamento com o adjuvante siliconado não diferiu significativamente do tratamento composto somente pelo herbicida, tendo um desempenho similar ao do tratamento com óleo mineral, que promove a redução da tensão superficial para valores próximos de $30 \mathrm{mN} \mathrm{m}^{-1}$, enquanto que a redução proporcionada pelo o adjuvante lecitina e ácido propiônico reduz a tensão superficial para valores em torno de 39 $\mathrm{mN} \mathrm{m}^{-1}$ (PRADO et al., 2016), evidenciando que, nas condições deste experimento, menores valores de tensão superficial da calda não promoveram a potencialização da fitotoxicidade do herbicida paraquate.

Os resultados expostos neste trabalho reforçam que a utilização de adjuvantes, na maioria dos casos, favoreceu a ação fitotóxica dos herbicidas clethodim e paraquat em plantas de capim-amargoso por fatores de interação variáveis segundo a classe do herbicida e do adjuvante. Os prováveis aspectos do favorecimento da ação fitotóxica relacionam-se com a facilitação promovida pelos adjuvantes na interação entre a calda e a superfície do vegetal, 
favorecendo o espalhamento, retenção, umectação e a absorção do ingrediente ativo do herbicida pela planta e a estabilização das propriedades físico-químicas da calda.

\section{Conclusão}

Conclui-se neste experimento que, o uso do herbicida clethodim em mistura com os adjuvantes nonil fenoxi poli (etilenoxi) etanol, lecitina e ácido propiônico e óleo mineral e o herbicida paraquat em mistura com o adjuvante lecitina e ácido propiônico, potencializou o efeito fitotóxico em plantas de capim-amargoso no estádio de florescimento.

\section{Agradecimentos}

Agradeço a Fundação de Amparo à Pesquisa do Estado de São Paulo (FAPESP) pela bolsa de iniciação científica concedida ao primeiro autor (Processo: 2017/00099-8).

\section{Referências}

BAIO, F. H. R.; GABRIEL, R. R. F.; CAMOLESE, H. S. Alteração das propriedades físico-químicas na aplicação contendo adjuvantes. Revista Brasileira de Engenharia de Biossistemas, v.9, n.2, p. 1-11, 2015.

https://doi.org/10.18011/bioeng2015v9n2p151-

161

CARVALHO, L. B. de; CRUZ-HIPOLITO, H.; GONZÁLES-TORRALVA, F.; ALVES, P. L. C. A.; CHRISTOFFOLETI, P. J.; De PRADO, R. Detection of sourgrass (Digitaria insularis) biotypes resistant to glyphosate in Brazil. Weed Science, v.59, n.2, p. 171-176, 2011. https://doi.org/10.1614/WS-D10-00113.1

CUNHA, J. P. A. R.; ALVES, G. S. Características físico-químicas de soluções aquosas com adjuvantes de uso agrícola. Interciência, v. 34, n. 9, p. 655-659, 2009.

CUNHA, J. P. A. R. da; ALVES, G. S.; MARQUES, R. S. Tensão superficial, potencial hidrogeniônico e condutividade elétrica de caldas de produtos fitossanitários e adjuvantes. Ciência Agronômica, v.48, n.2, p. 261-270, 2017.

DEER, H. M.; BEARD, R. Effect of water pH on the chemical stability of pesticides. Electronic report AG/Pesticides/14 of Utah State University Extension, v.14, n.7, p. 1-3, 2001.
DORR, G. J.; FORSTER, W. A.; MAYO, L. C.; MCCUE, S. W.; KEMPTHORNE, D. M.; HANAN, J.; TURNER, I. W.; BELWARD, J. A.; YOUNG, J.; ZABKIEWICZ, J. A. Spray retention on whole plants: modelling, simulations and experiments. Crop Protection, v.88, p. 118-130, 2016. https://doi.org/10.1016/i.cropro.2016.06.003

HOLLOWAY, P. J.; BUTLER ELLIS, M. C.; WEBB, D. A.; WESTERN, N. M.; TUCK, C. R.; HAYES, A. L.; MILLER, P. C. H. Effects of some agricultural tankmix adjuvants on the deposition efficiency of aqueous sprays on foliage. Crop Protection, v.19, n.1, p. 27-37, 2000.

https://doi.org/10.1016/S0261-2194(99)00079-4

JORDAN, D. L.; VIDRINE, P. R.; GRIFFIN, J. L.; REYNOLDS, D. B. Influence of adjuvants on efficacy of clethodim. Weed Technology, v.10, n.4, p. 738-743, 1996.

https://doi.org/10.1017/S0890037X00040744

LI J.; CHEN, W.; XU, Y.; WU, X. Comparative effects of different types of tank-mixed adjuvants on the efficacy, absorption and translocation of cyhalofop-butyl in barnyardgrass (Echinochloa crus-galli [L.] Beauv.). Weed Biology and Management, v.16, n.2, p. 80-89, 2016. https://doi.org/10.1111/wbm.12095

LOPEZ-OVEJERO R. F; TAKANO H. K.; NICOLAI M.; FERREIRA A.; MELO M. S. C.; CAVENAGHI A. L.; CHRISTOFFOLETI P. J.; OLIVEIRA J. R. Frequency and dispersal of glyphosate-resistant sourgrass (Digitaria insularis) populations across brazilian agricultura Iproduction areas. Weed Science, v. 65, n.2, p. 285-294, 2017.

https://doi.org/10.1017/wsc.2016.31

MACHADO, A. F. L.; FERREIRA, L. R.; FIALHO, C.M.T. Análise de crescimento de Digitaria insularis. Planta Daninha, v.24, n.4, p. 641-647, 2006.

http://dx.doi.org/10.1590/S01008358200600040 $\underline{0004}$

MACHADO, A. F. L.; MEIRA, R. M. S.; FERREIRA, L. R.; FERREIRA, F. A.; TUFFI SANTOS, L. D.; FIALHO, C. M. T.; MACHADO, M. S. Caracterização anatômica de folha, colmo e rizoma de Digitaria insularis (L.) Fedde. Planta Daninha, v.26, n.1, p. 1-8, 2008. http://dx.doi.org/10.1590/S0100$\underline{83582008000100001}$ 
MASSINON M.; BOUKHALFA H.; LEBEAU F. The effect of surface orientation on spray retention. Precision Agriculture, v.15, n.3, p. 241-25, 2014. https://doi.org/10.1007/s11119-013-9345-2

MELO, M. S. C.; ROSA, L. E.; BRUNHARO, C. A. C. G.; NICOLAI, M.; CHRISTOFFOLETI, P. J. Alternativas para o controle químico de capimamargoso (Digitaria insularis) resistente ao glyphosate. Revista Brasileira de Herbicidas, v.11, n.2, p. 195-203, 2012.

https://doi.org/10.7824/rbh.v11i2.145

NANDULA, V. K.; POSTON, D. H.; REDDY, K. N.; KOGER, C. H. Formulation and adjuvant effects on uptake and translocation of clethodim in bermudagrass (Cynodon dactylon). Weed Science. v.55, n.1, p. 6-11, 2007.

https://doi.org/10.1614/WS-06-024.1

PEREIRA, M. C.; ESPANHOL, M.; DUARTE, D. J.; CORRE, N. M. Manejo químico de Digitaria insularis em área de plantio direto. Revista Brasileira de Ciências Agrárias, v.5, n.1, 2010. https://doi.org/10.5039/agraria.v5i1a481

PRADO, E. P., RAETANO, C. G., DO AMARAL DAL, M. H. F., CHECHETTO, R. G., FERREIRA FILHO, P. J. MAGALHAES, A. C., \& MIASAKI, C. T. Effects of agricultural spray adjuvants in surface tension reduction and spray retention on Eucalyptus leaves. African Journal of Agricultural Research, v.11, n.40, p. 3959-3965, 2016. https://doi.org/10.5897/AJAR2016.11349

RITZ, C.; STREIBIG, J. C. Dose response curves and other nonlinear curves in Weed Science and Ecotoxicology with the add-on package drc in R. PLoS One 10, C0146021, 2015.

RITZ C.; BATY F.; STREIBIG J. C.; GERHARD D. Dose-response analysis using R. PLoS One, v.10, n. 12, 2015.

https://doi.org/10.1371/journal.pone.014602

RYCKAERT B.; SPANOGHE P.; HAESAERT G.; HEREMANS B.; ISEBAERT S.; STEURBAUT $W$. Quantitative determination of the influence of adjuvants on foliar fungicide residues. Crop Protection, v.26, n.10, p. 1589-1594. 2007. https://doi.org/10.1016/i.cropro.2007.02.011

SALVALAGGIO A. C.; Da COSTA, N. V.; CAZZO, V. N.; CONRADI JUNIOR, E.; ECKERT A. F.
Espalhamento e tensão superficial de gotas de soluções com herbicidas e adjuvantes em folhas de Digitaria insularis. Journal of Neotropical Agriculture, v.5, n.3, p. 60-64. 2018.

https://doi.org/10.32404/rean.v5i3.1721

SEEFELDT, S. S.; JENSEN, J. E.; FUERST, E. P. Loglogistic analysis of herbicide dose response relationship. Weed Technology, v.9, n.2, p. 218227, 1995. https://doi.org/10.1017/S089

SOCIEDADE BRASILEIRA DA CIÊNCIA DAS PLANTAS DANINHAS (SBCPD). Procedimentos para instalação, avaliação e análise de experimentos com herbicidas. Londrina: SBCPD, 1995. $42 \mathrm{p}$.

VARGAS, L.; ROMAN, E. S. Conceitos e aplicações dos adjuvantes. Passo Fundo: Embrapa Trigo, 2006. 10 p. (Embrapa Trigo. Documentos Online, n. 56).

ZOBIOLE, L. H. S.; KRENCHINSKI, F. H.; ALBRECHT, A. J. P.; PEREIRA, G.; LUCIO, F. R.; RUBIN, R. S. Controle de capim-amargoso perenizado em pleno florescimento. Revista Brasileirade Herbicidas, v.15, n.2, p. 157-164, 2016. https://doi.org/10.7824/rbh.v15i2.474 